ISAHP 1999, Kobe, Japan, August 12-14, 1999

\title{
INTELLIGENT SOFTWARE BASED ON AHP
}

\author{
Andreichicov A.V. and Andreichicova O.N. \\ Department of Information Systems in Economics, Volgograd State Technical University \\ Department of Computers and Networks, Volgograd State Technical University \\ Volgograd, 400066, Lenin avenue, 28, Russia \\ Phone: (+7 8442) 97-91-70 Fax: (+7 8442) 97-74-70 E-mail: andr@ystu.ru
}

\begin{abstract}
This paper describes the developed intelligent software for decisions support, based on AHP. Such a system is able to solve static and dynamic decisions making problems and it's able to carry out a consequences forecasting of the accepted decisions. Described decision support system (DSS) includes a database, a knowledge base and a subsystem for knowledge extraction from database (data-mining system). The database contains the information, received from the experts during the problems' solving. The knowledge base is filled with the information, which is formed on the base of the analysis of solved problems. The developed software has the friendly user interface. The example of the solving dynamic problem with use of the developed software are considered.
\end{abstract}

\section{Introduction}

The decisions making problem (DMP) takes place when it is necessary to make a choice of the best variant among a given set of alternatives. Any choice is connected with an information processing about alternatives, about quality criteria, about probable outcomes, about preferences systems and about the ways of a mapping of a admissible alternatives' set in a set of the criteria estimations of probable outcomes. Depending on a kind of such mapping DMP may be subdivided into the following categories:

- The problems in conditions of certainty, which are characterised by the complete and exact initial information and by determined type of the mapping of the aiternatives' set in a set of criteria estimations, i.e. the adequate mathematical description of a problem is available. For the solution of such problems mathematical programming methods are usually applied;

- The problems in conditions of risk take place in the case when the probable outcomes can be described with help of some probability distribution. Usually it is necessary to have statistical or expert data for a building of such distribution. For a solving of these problems the methods of the utility theory are often applied;

- The problems in conditions of uncertainty take place, when the initial information is incomplete, inexact and the type of a formal mapping either is too complex, or is not known. In such cases the experts' knowledge are attracted for the DMP solving. There are different approaches to the representation and the processing of these knowledge based on various methods of the applied decision theory and on the AI methods.

The mathematical methods of the decision theory are based on a knowledge representation as some quantitative data, being estimations of experts' preferences. The methods differ by the manners of representation and processing of preferences and frequently result in different outcomes. In this connection there is a problem of a choice of the strategy and the method for a particular problem solving. The criteria for a method choice in each case will depend on quantity and quality of the accessible information, of accepted problem statement and of problem's environment. There are the problems, rather indifferent to environment parameters' change and on the contrary; the changes of these parameters can have various character (smooth, sharp, qualitative and etc.). Therefore mathematical approach to DMP include difficult problems of a method choice a and substantiation of received results. 
The approach to decisions making from positions of artificial intelligence essentially differs from above mentioned. The expert systems carry out decision support also, but they use quite different strategy of the problems solving. DSS are intended for the user - expert, but expert systems - for user-inexpert, as they already incorporates experts knowledge. The expert system is less flexible in comparison with DSS, as the structure of a knowledge base and accepted inference engine impose certain restrictions on the soluble problems. Besides that the development of self-training expert systems filled by incomplete and inexact knowledge consumes much time, therefore this approach is poorly acceptable to quickly developing knowledge fields.

The subject of further consideration are the systems for computer support of dynamic DMP in conditions of uncertainty. Dynamic DMP are characterised by unstable in time initial information, i.e. there are changes of a structure and properties of alternatives, of a set of choice criteria, their relative gravity and expert preferences too. In dynamic DMP all categories of the information are subject to changes, thus a kind of expert preferences' change reflects the tendencies of changes, occurring in a problem environment. These tendencies can be estimated on the basis of stored statistics: Therefore the dynamic DMP in conditions of uncertainty demand the attraction, accumulation and multivariate processing of large volumes of the expert information. Such information can be used for forecasting of considered variants' preferences, for an estimation of probable consequences of the accepted decisions and reception of new knowledge in researched area.

\section{The description of intelligent DSS}

The present paper discusses DSS for dynamic processes of the decisions making in conditions of uncertainty, incorporating a database, a knowledge base, a mathematical methods block, a data-mining subsystem, a multifunctional user interface. The developed DSS is based on AHP, its development and adaptation for achievement of the following aims:

- The support of the process of a multicriterial multialternate choice, carried out on the base of expert estimations with use of the information from a database.

- Providing user with the friendly interface and formation of the solving problem's protocol, which is a detailed substantiation of the outcomes;

- Building of a database, where initial information and results of the solved problems, relating to particular knowledge fields, are stored. Filling the database occurs during the solving of DMP in course of time;

- Providing the support of collective decision making;

- Estimation of the nearest consequences of the accepted decisions based on a forecasting of experts preferences, of probable changes of criteria priorities and alternatives, of established trends of gravity of the factors, criteria and etc.;

- Reception of new knowledge based on a processing the information, stored in a database;

The mathematical part of DSS includes the following AHP procedures (Saaty, 1990): check of consistency of pair comparison matrixes, computation of normalised eigenvector for pair comparison matrixes, computation of a priorities vector for quantitative measured alternatives; computation of a priorities vector for alternatives, measured with the help of the linguistic standards; calculation of marginal priorities vectors; the linear convolution on a hierarchy of criteria, including incomplete hierarchy (when criteria are connected with various subsets of alternatives). For the solution of practical problems with dynamic judgements we have included in DSS the following procedures (Andreichicov and Andreichicova, 1997,1998):

- Selection of functions and building of polynomials, which approximates changes of preferences and priorities on the base of the information, stored in a database;

- Computation of an eigenvector of a dynamic pair comparisons matrix of arbitrary dimension.

The database in DSS carries out two basic functions. It is used for information user support at the formation of new problems in the given knowledge field, and also during data-mining processes. The 
knowledge representation about the goals, actors, criteria, alternatives, experts and preferences is complex, as there are a lot of various types' connections between the data elements. Data may be divided into two categories according to the basic functions. The first group provides information user support and the second group, more particular, is used during data-mining. The information on alternatives, criteria and experts relates to the first category. The second category of data contains the references to particular alternatives, criterion and experts, participating in the solution of a certain problem, and includes the information on preferences and priorities. The first category's data may be represented by every data model, but the second category's data are poorly described by any models. There was produced a special format for their representation, named a problem's file (Andreichicov and Andreichicova, 1998). The scheme of a database structure is shown on fig. 1 .

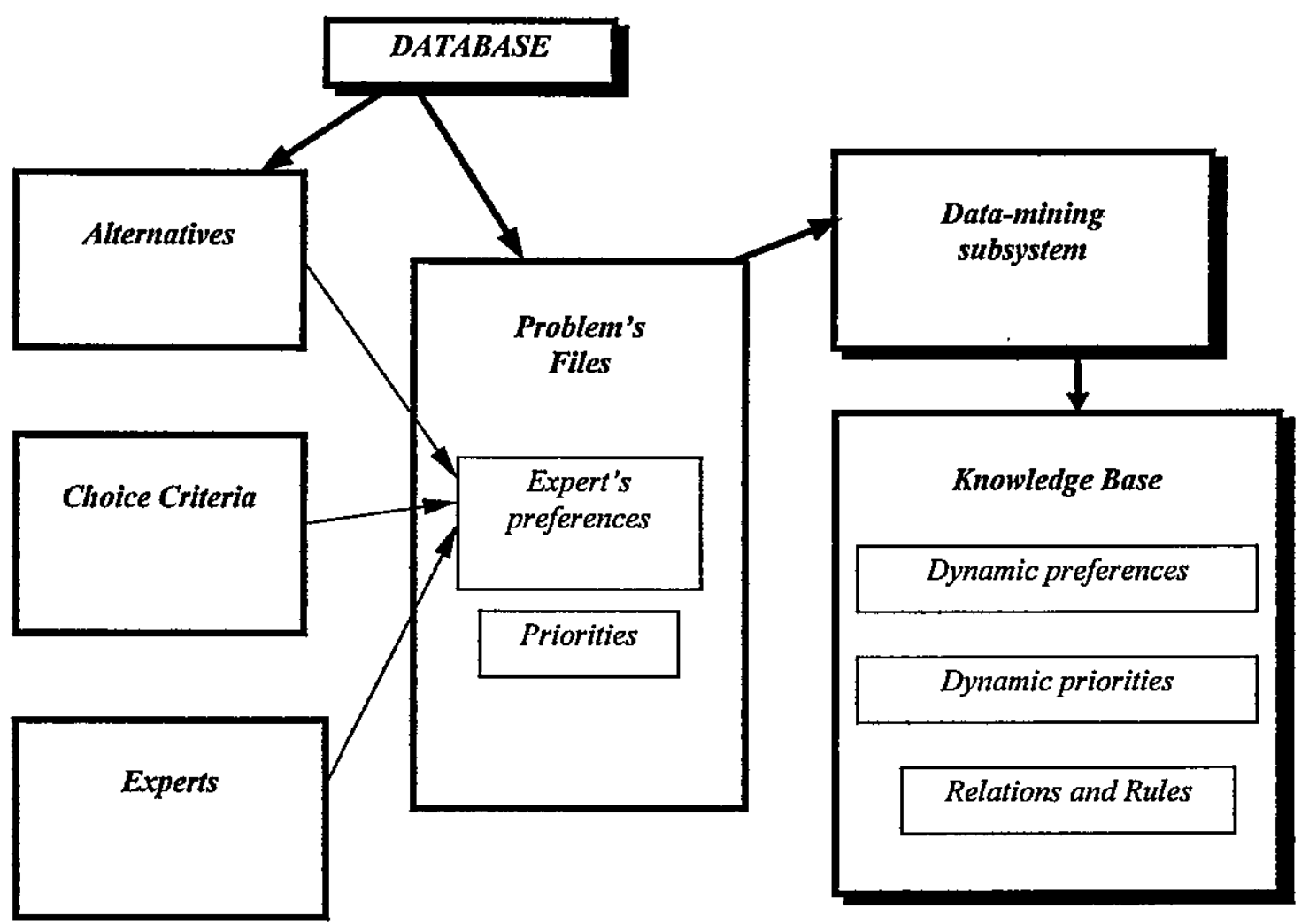

Figure 1. The database structure scheme.

The account of the various experts' judgements is carried out by the calculation of geometrical average judgement, thus the separate judgements should not be contrary. In the case of opposite judgements should both solve a problem for each expert separately, or find the compromise at the stage of preparation of the information.

The consequences estimation of the accepted decisions is made on a base of the marginal analysis, of the dynamic problems' solution and of the statistical forecasting. A forecasting of the preferences and priorities with the help of approximate functions is possible for the problems, which have statistics saved in a database. During a dynamic DMP solving, one should receive associations, which describe priorities changes in course of time on the base of the information on dynamic experts judgements. That is, the expert should estimate not only preference of an alternative before other, but also probable change of this estimation for a certain time interval. If authentic statistics is absent it is possible to carry out expert estimation of probable preferences change by a functional scale (Saaty, 1990). 
The screen forms of developed DSS are brought in fig. $2-3$. There is the item of Knowledge in the main menu, which opens the opportunities for the statistical information analysis and for a search of the main tendencies and laws of the information changes. The different means for making representative samples from problems' files are given to the user, they are based on use of various similarity measures. Received samples are employed for a building of the regression functions, which describe change of preferences and priorities in course of time. Besides that, there are the opportunities for the realising of the frequencies analysis of criteria and alternatives in problems and for revelation of promising alternatives in future. Equipment of DSS by data-mining methods enables to establish the relations between various information categories (between criteria, actors, goals, factors etc.). A such results are new knowledge. It is necessary to choose the appropriate format for representation of this knowledge. In discussed realisation of DSS for knowledge representation the objects (frames) and production rules are used. The frame approach enables correctly to organise and to process functional associations being received. Productions rules usually describe the ideas of an optimum alternatives set and the relations of choice criteria (Andreichicov and Andreichicova, 1997,1998).

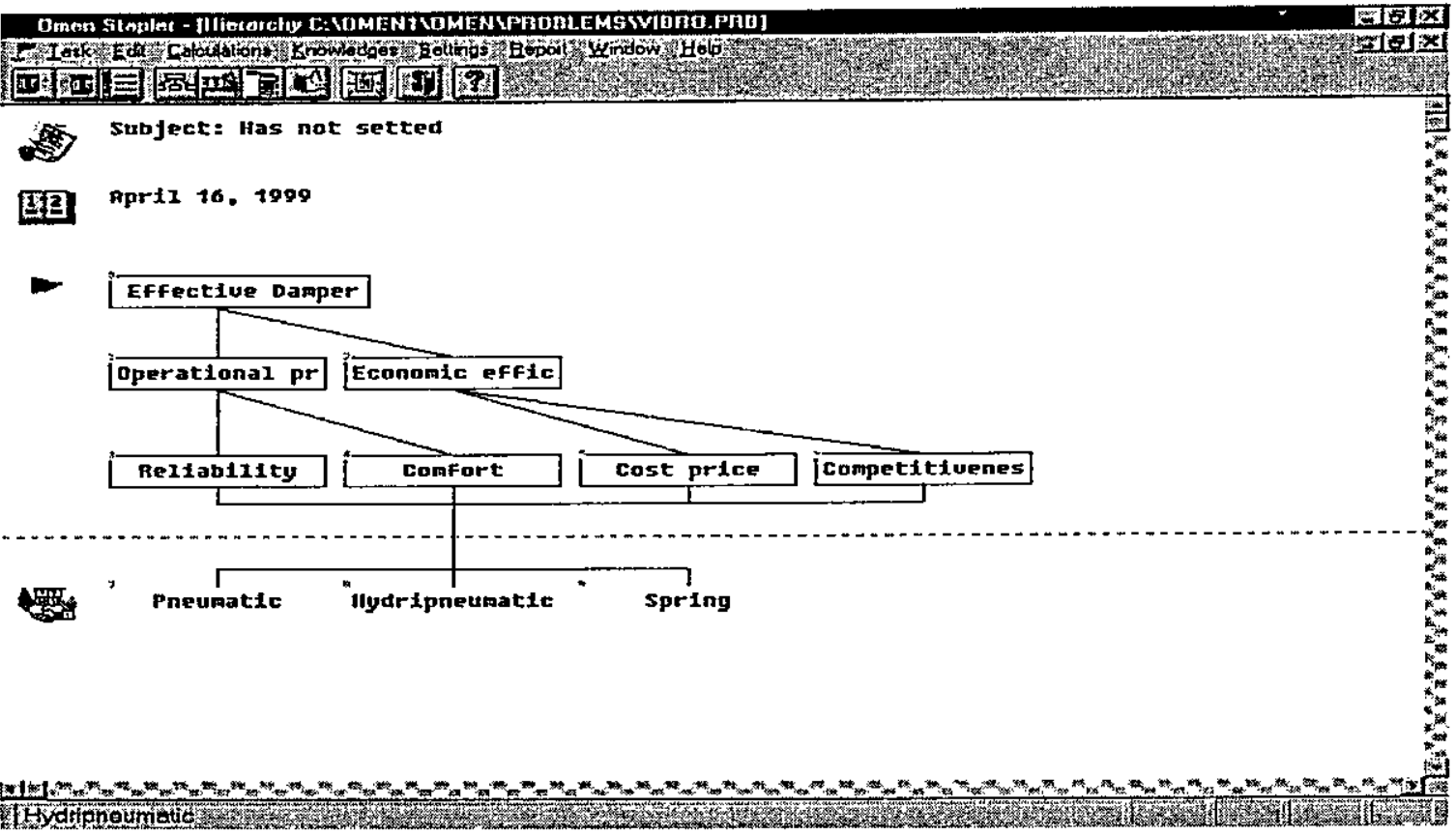

Figure 2. Screen form for criteria hierarchy.

\section{Example of dynamic problem solving}

Let's consider a problem of shock-absorber efficiency forecasting for 5 years. The quality criteria hierarchy is shown on fig. 2. Alternatives are the snubbers for transport machines: pneumatic, hydropneumatic and spring. The expert preferences are represented by functions, which are brought in tab. 1, 2. The outcomes of the solved problem are shown on fig. 4 . The best variant is hydropneumatic snubber, its priority will increase in course of time. Pneumatic snubber priority wavy will decrease in time, and spring priority linearly will decrease. 


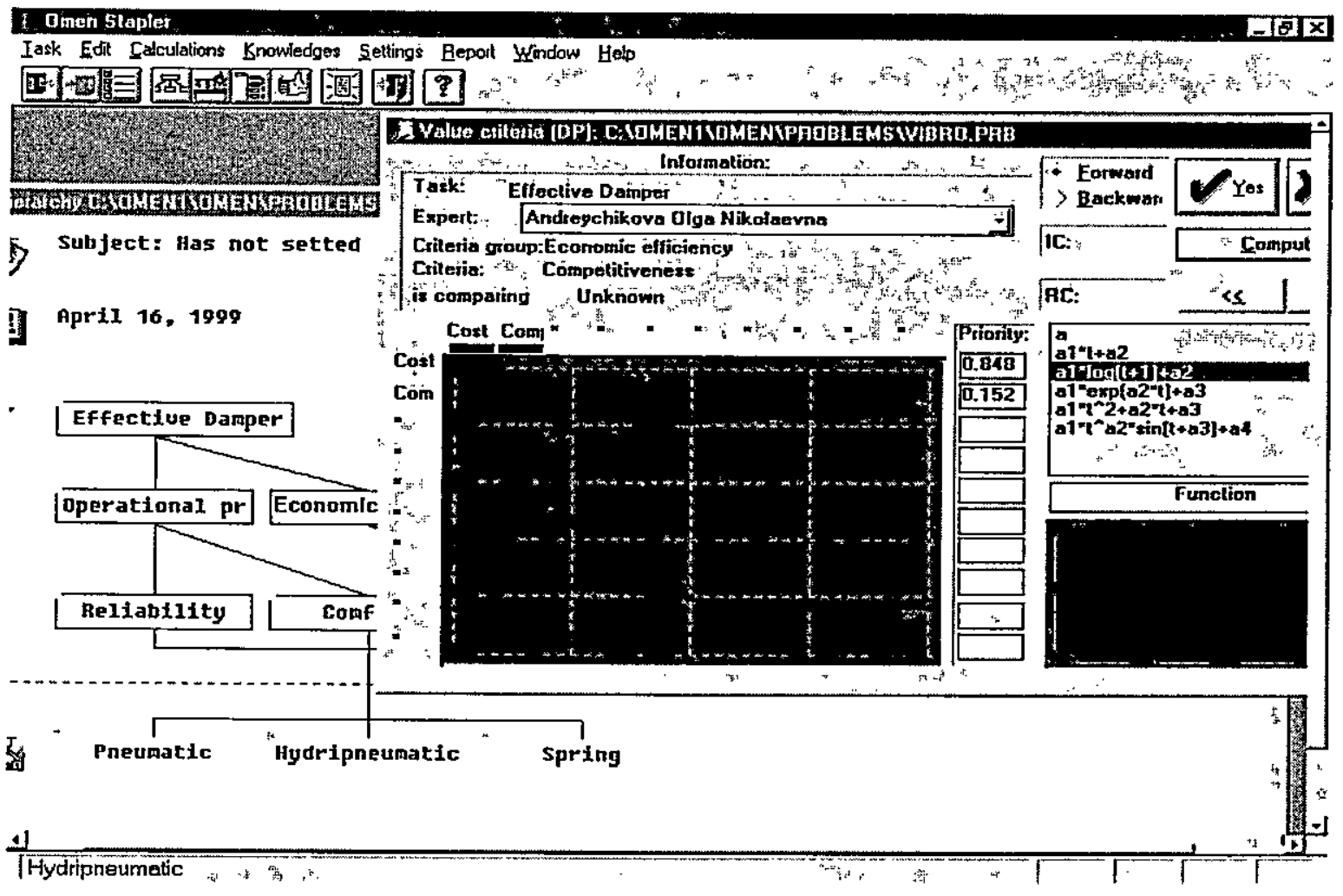

Figure 3. Screen at dynamic problem solving.

Table 1. Dynamic judgements for the criteria set.

\begin{tabular}{|c|c|c|c|}
\hline Reliability & Pneumatic & Hydropneumatic & Spring \\
\hline $\begin{array}{c}\text { Pneumatic } \\
\text { Hydropneumatic } \\
\text { Spring }\end{array}$ & $\begin{array}{l}y_{11}=1 \\
y_{21}=0.01 \cdot e^{t .1 t}+2 \\
y_{31}=0.5 t+5\end{array}$ & $\begin{array}{l}y_{12}=1 / y_{21} \\
y_{22}=1 \\
y_{32}=-0.5 t+3\end{array}$ & $\begin{array}{l}y_{13}=1 / y_{31} \\
y_{23}=1 / y_{32} \\
y_{33}=1\end{array}$ \\
\hline Comfort & Pneumatic & Hydropneumatic & Spring \\
\hline $\begin{array}{c}\text { Pneumatic } \\
\text { Hydropneumatic } \\
\text { Coil spring }\end{array}$ & $\begin{array}{l}y_{11}=1 \\
y_{21}=0.01 e^{1 . t t}+3 \\
y_{31}=1 / y_{13}\end{array}$ & $\begin{array}{l}y_{12}=1 / y_{21} \\
y_{22}=1 \\
y_{32}=1 / y_{23}\end{array}$ & $\begin{array}{l}y_{13}=1.0 \cdot \log (t+1)+3 \\
y_{23}=1.0 \log (t+1)+5 \\
y_{33}=1\end{array}$ \\
\hline Cost price & Pneumatic & Hydropneumatic & Spring \\
\hline $\begin{array}{l}\text { Pneumatic } \\
\text { Hydropneumatic } \\
\text { Coil spring }\end{array}$ & $\begin{array}{l}y_{11}=1 \\
y_{21}=1 / y_{12} \\
y_{31}=1 / y_{13}\end{array}$ & $\begin{array}{l}y_{12}=3.8 \log (t+1)+3 \\
y_{22}=1 \\
y_{32}=-0.5 t+7\end{array}$ & $\begin{array}{l}y_{13}=0.4 t+3.0 \\
y_{23}=1 / y_{32} \\
y_{33}=1\end{array}$ \\
\hline Competitiveness & Pneumatic & Hydropneumatic & Spring \\
\hline $\begin{array}{l}\text { Pneumatic } \\
\text { Hydropneumatic } \\
\text { Coil spring }\end{array}$ & $\begin{array}{l}y_{11}=1 \\
y_{21}=1.0 t+3 \\
y_{31}=1 / y_{13}\end{array}$ & $\begin{array}{l}y_{12}=1 / y_{21} \\
y_{22}=1 \\
y_{32}=1 / y_{23}\end{array}$ & $\begin{array}{l}y_{13}=0.25 t^{2}+0.5 t+1 \\
y_{23}=1.00 t^{27} \cdot \sin (t+1)+5 \\
y_{33}=1\end{array}$ \\
\hline
\end{tabular}


Table 2. Dynamic judgements for alternatives.

\begin{tabular}{|c|c|c|}
\hline Effective Shock-absorber & Operational properties & Economic efficiency \\
\hline $\begin{array}{l}\text { Operational properties } \\
\text { Economic efficiency }\end{array}$ & $\begin{array}{l}y_{11}=1 \\
y_{2 I}=I / y_{12}\end{array}$ & $\begin{array}{l}y_{12}=0.01 e^{1.3 t}+1 \\
y_{22}=1\end{array}$ \\
\hline Operational properties & Reliability & Comfort \\
\hline $\begin{array}{l}\text { Reliability } \\
\text { Comfort }\end{array}$ & $\begin{array}{l}y_{11}=1 \\
y_{21}=3.5 \log (t+1)+1\end{array}$ & $\begin{array}{l}y_{12}=1 / y_{21} \\
y_{22}=1\end{array}$ \\
\hline Economic efficiency & Cost price & Competitiveness \\
\hline $\begin{array}{c}\text { Cost price } \\
\text { Competitiveness }\end{array}$ & $\begin{array}{l}y_{11}=1 \\
y_{21}=t+1\end{array}$ & $\begin{array}{l}y_{12}=1 / y_{21} \\
y_{22}=1\end{array}$ \\
\hline
\end{tabular}

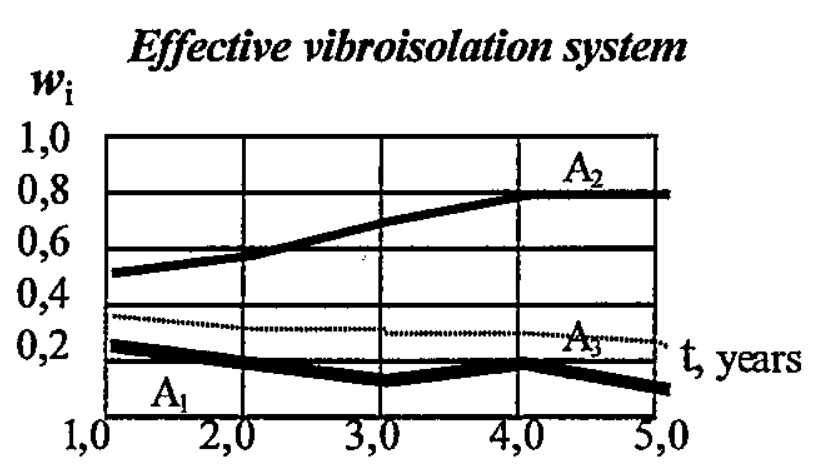

Figure 4. Resulting priorities for alternatives: $\mathbf{A}_{\mathbf{1}}$ - pneumatic; $\mathbf{A}_{2}$-hydropneumatic; $\mathbf{A}_{3}$-spring.

\section{Conclusion}

AHP realises the rational - weighed approach to a decisions making problem. Such approach is suitable for many problems in various knowledge fields. The simple mathematics and the clear strategy of the problems solving make AHP accessible for understanding of inspecialists. AHP has wide prospects for development and application for problems of planning, forecasting etc. Main lacks of this approach are connected with restriction on amount of compared alternatives and with long-time procedures of pair comparison. In our sight, overcoming of the specified problems and the expansion of AHP opportunities are possible by creating of intelligent software, based on this method.

\section{References}

Saaty T.L. (1990) The Analytic Hierarchy Process, RWS Publications, Pittsburgh, PA.

Andreichicov A.V. and Andreichicova O.N. (1998) Computer Support of Invention (methods, systems, cases), Machine Building, Moscow.

Andreichicov A.V. and Andreichicova O.N. (1997) "Computer support of invention processes", Proceedings of the 11th International Conference on Engineering Design in Tampere, Vol. 2, Schriftenreihe WDK 25, Tampere, 197-202. 